\title{
A Review of various Face Prediction Models using Image Processing
}

\author{
Nitesh Kumar \\ Department of Computer \\ Science Engineering \& \\ Technology \\ Amity University, Haryana
}

\author{
Vivek Jaglan, PhD \\ Assistant Professor \\ Department of Computer \\ Science Engineering \& \\ Technology \\ Amity University, Haryana
}

\author{
Akshat Agrawal \\ Assistant Professor \\ Department of Computer \\ Science Engineering \& \\ Technology \\ Amity University, Haryana
}

\begin{abstract}
Out of all body parts face is one of the most important part of body by which everyone can show its emotions, feelings etc. Most of the humans can easily predict a person's current age just by gazing their faces. Facial recognition is a part of biometric software application which is used to identify a particular and individual thing in an image by analysis and evaluation of patterns. There are various face prediction models which are based on different techniques like PCA, ANN etc. Age plays an important role to predict the face of any person. Most of the models are built on the basis of age parameter. Recently, image processing has played a major role in this area of research and has widely used for the face prediction. These ages based models have various useful applications like security purpose, to find a missing person. This paper presents a survey of various face prediction systems using image processing techniques in recent times. A comprehensive study of a number of face prediction systems are done in this paper, with different methodologies and their performances.
\end{abstract}

\section{Keywords}

Face prediction, PCA, ANN, image processing, face recognition.

\section{INTRODUCTION}

A very powerful medium of human communication is human face [4], carrying lots of information about the person like emotional state, identity, gender, ethnicity. Simulation of face age and prediction of face is a recent research area with numerous applications in digital entertainment. The main goal for all applications is to produce aging effects that are generally possible without changing the identity of a person. This is a different task from face recognition in biometrics because in this task two key assumptions are consider that extract those features which are constant over a long time period and evaluate the rate of inclination to which exterior look of face is changed in aging process. A logical model is needed to evaluate and analyze the sequel of aging on faces. Effectiveness and efficiency of automated face recognition system is greatly affected by the aging of faces. Light and photograph difference are other important factors to reduce the efficiency of face recognition system. Various face recognition models are divided into space and frequency domain on the basis of various algorithms. In space domain, geometric and Template Matching using Principle Components Analysis, Independent Component Analysis, Linear Discriminant Analysis are generally used methods.DCT(Discrete Cosine Transforms) and Gabor Wavelet are generally used methods for frequency domain .As humans, man got complete command over human morphological genetics, face of a person totally depend upon natural features that are opulent and sturdy lines, unswerving nose, potent eyes, location of facial features, and left or right perfect symmetry that plays an important role. It is expected that human face is keep changing as time spent .In current time the size of human brain is three times the size of inherent ancestors. Head get bigger with the growth of brain which leads to the expansion of skull causing facial feature to be more flat.

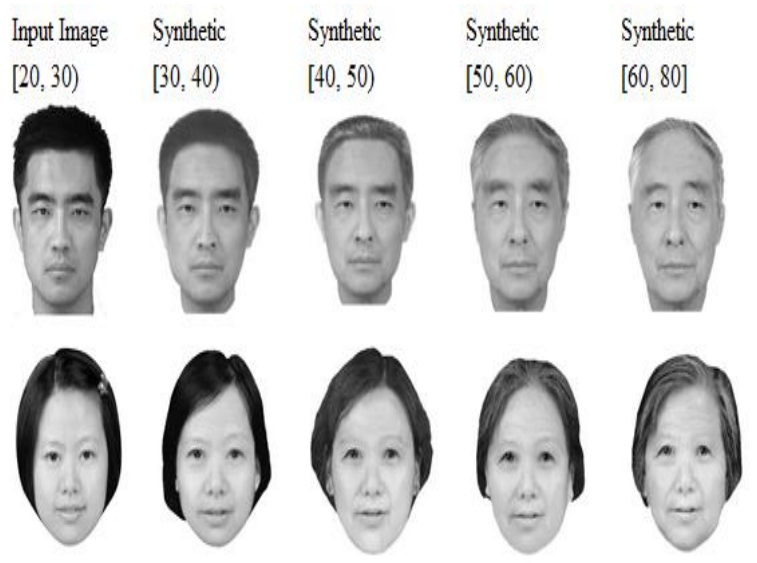

Fig.1: images of face prediction

\subsection{Requirements for face prediction [4]}

Following are the universal requirements for predicting the face .To get detailed information; we have to knowledge about these parameters:

1. Essential: For essential or invariant qualities face performs as an index like sex and race. Lots of researches show that Americans has intelligence by birth, while other culture looks it as socially reliant.

2. Biologic Antiquity: A person's face shows its biological Antiquity, by virtue of which it can make judgment of some qualities like person's age, health, and energy level, are found to be most important dimensions for predicting future performance.

3. Moral Antiquity: Frequent occurrence of intention and emotion might leave spot onto the face of a person which produce qualities like learned kindness, generosity and honesty.

4. Social History: From the human face, anyone can easily read a person's social history which produce qualities like grooming level, wealth, confidence and dominance. 
5. Professional History: Professional history can produce qualities like experience, competence and trustworthiness.

\section{LITERATURE SURVEY \\ 2.1 Face Prediction Model for an Automatic Age-invariant Face Recognition System [1]}

Automated face recognition and identification systems plays important role in quotidian life. Its scope is not limited up to Facebook auto photo tagging, iPhoto by apple, Picasa by google and Kinect by Microsoft's. It is also used in Homeland Security Department's, dedicated biometric face detection systems, to find a missing person. The main challenging task is to how to handle facial changes during reasonable conditions like natural aging. It is still an unsolved problem. Individual face components for e.g. eyes, nose, mouth features Based matching algorithms has been developed but these are not efficient algorithms. Therefore, a Face Prediction Model (FPM) is developed, which is based on Principle Component Analysis and Artificial Neural Network learning methods and this model is used to predict face age and effect of growth in an image. Firstly, FPM is used to capture the facial changes, which occur due to human aging and predicts the facial image with a few years of gap. The accuracy of this model with of face matching lies in between from 76 to $86 \%$. The time series image data is required by the FPM. This data is used to calculate the temporal and spatial correlation between the images to predict the next time series image.

\section{Steps to implement the proposed system:}

1) Capture time series face images.

2) Apply image processing.

3) Normalization.

4) Apply Short Term Fourier Transformation (STFT).

5) Apply Principal Component Analysis to extract the facial features from the result of STFT.

6) Train and test ANN (Artificial Neural Network).

7) Output predicted image of face.

This is a useful model implemented in MATLAB for face prediction with some computational effort.

\subsection{Subjective Age Prediction of Face Images Using PCA [2]}

As humans anyone may accurately predict a person's current age just by gazing their faces. It can happen by reading variations onto their face like wrinkles. Age prediction is very easy task for humans but it is tough and time consuming for computers. Age is an important factor for predicting any human face. There are lots of face prediction models has been implemented but no model gives accurate and efficient result. In this paper, Principal Component Analysis technique is used to predict age of human. Another name for principal component analysis is Karhunen-Loève expansion. It is a popular technique used for features extraction and to represent data. That's why it is widely used in the research area of pattern recognition and computer vision. On the basis of PCA, features are extracted from any image and these useful features are further used to predict face of a person. Facial database is built by means of seven discrete age classes which are ranging from the face images of adults between 10 to 60 years old. To identify the age of a person an age prediction algorithm is used to predict age. In this model, Age prediction is done by training the system using training set that is capable of predicting the ages of the facial images. Training set is constructed using geometric and principal component analysis facial features extracted from an image. The accuracy of the system is examined by the contrast in the span of the age classes. System efficiency can be analyzed through the experiment outcomes.

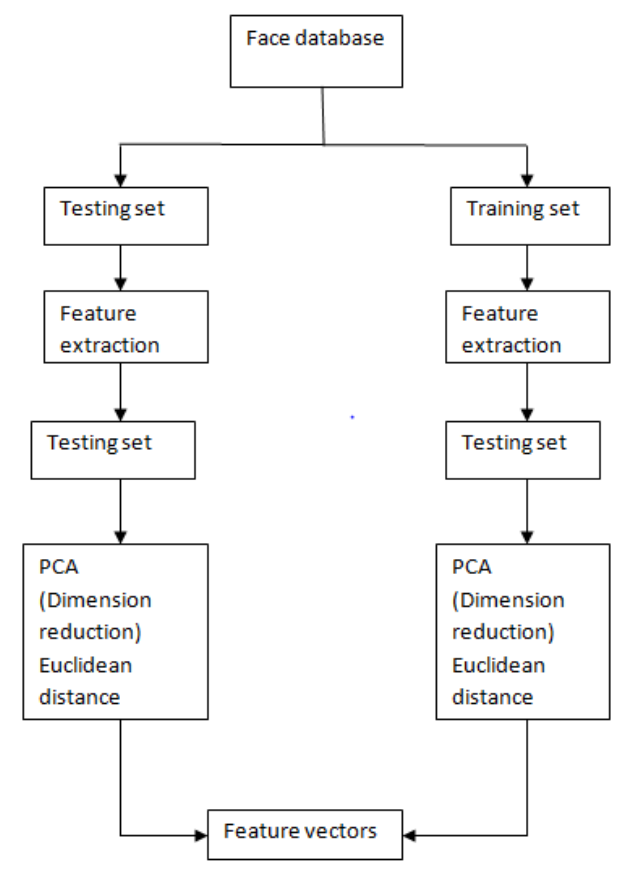

Fig 2: flow chart of human face age prediction system

This model has total seven groups named from group 1 to group 7 . The accuracy rate for group 1, group 2 and group 7 is $100 \%$. For group 3, correct accuracy rate is $90.5 \%$. For group 4 , group 5and group 7 it is $91.5 \%, 93.5 \%, 94.5 \%$. Overall prediction rate for 1300 images is found to be $92.5 \%$. It can be inferred that the performance of system is $92.5 \%$ in age prediction.

\subsection{Facial Image Prediction Using Exemplar-based Algorithm and Non- negative Matrix Factorization [3]}

There is various range of application for Human face prediction e.g. Finding a person who was gone missing from a long period of time and for the analyzing the output of change of face in medicines etc. This paper mainly proposed Exemplar method base algorithm whose property is to take in consideration the sequel of human growth. This models based on two methods that are first one is non-negative matrix factorization and secondly linear interpolation techniques. These techniques are used for predicting the facial images. They primarily considered six facial Region of Interest (ROI) of an image that is nose, eye-brow, eye, and mouth. For this model, only those images of family are considered in whichever member has found to have more than one image at different age. Based on these images predicts the image Region of Interests that are been replaced with the actual image to obtain the prediction outcomes. Key problem is to assemble families face images Region of Interests at distinct age. Database build from the internet is used in this model. Outcome of experiment result shows that the correlation coefficient between the realistic and predicted image found up 
to 0.82 . Correlation factor is affected by expressions and light in the reference images.

\section{Steps to implement the proposed system}

1) Take an image.

2) Identify similarity determination.

3) Apply NMF (Non-negative Matrix Factorization) based scheme.

4) Identify predicted Region of Interests.

5) Perform Face synthesis.

\section{Database construction}

1) Store face images with age information.

2) Extract Region of Interests from face image.

3) Prepare database.

This model is developed by using Visual $\mathrm{C}++$ and Microsoft office methods. Experiment outcomes are divided in three parts: premise images, sequence of weight interpolations images, and sequence of region of interest's synthesis. This model is basically used to extract six regions of interests from facial image instead of extracting whole face.

\subsection{Automated Facial Trait Judgment and Election Outcome Prediction: Social Dimensions of Face [4]}

For human communication and to acquire a significant resource of information, human face plays the most important medium. It is also a source to gather many other essential traits. This paper is based on a fully automated face recognition system that can assume apparent qualities of a person from his facial social aspects like brilliance, morals, competence, and relates how those qualities can serve to predict outcomes of real-time social occurrence that consider long-term fidelities like political election, job hiring, and marriages. A hierarchical system is developed to identify apparent quality face, incorporation of top-level perception and intermediate features. Developed model is used for classification of the outcomes of two most important political occasions by utilizing the facial image of politician. Primarily, it used classify the victors of a series of recent United States ballot. Accuracy of the proposed system outcomes is found to be $67.9 \%$ for prediction of the Governors and $65.5 \%$ for predicting the Senators. Distinct qualities are preferred by distinct politicians. Secondarily, purposed model can splits the politician's political alliance amalgamations i.e. Democrat alliance vs. Republican alliance. Precision of the system for predicting male is found to be $62.6 \%$ and for female it is about $60.1 \%$. This model is found to reasonably scalable; also it has more objectives as compared to previous models and provides vast range of contemporary applications.

\subsection{A Compositional and Dynamic Model for Face Aging [5]}

This paper is based on a developing a system model that can compositionally and dynamically models face aging. A hierarchical AND-OR graph technique is used for the developing compositional modeling and it is used to predict faces for different age groups. In this model, AND nodes are used to divide a face into pieces to define information like hair, wrinkles etc. OR nodes are used to show broad scope of different selected faces. An AND-OR parse graph traversal is an instance of face. Aging of face is elucidated as Markov process on the parse graph delineation. A big annotated face database is prepared and on the basis of this database, important parameters for this model are found and the dynamics explicitly elucidate random facial aging. By virtue of this model, an algorithm is developed that dynamically predict and simulate face aging and called automatic age estimation algorithm. On the basis of this dynamic model, two important criteria's are found to analyze the outcome of aging using experiments on human discernment. Firstly it focuses on simulation accuracy which is defined as whether the aged faces are discerned of the deliberate age class. Secondly it focuses on identity preservation which is defined as whether the aged faces is discerned as the concordant person. The performance of this model is analyzed by Quantitative statistical analysis and age estimation algorithm.

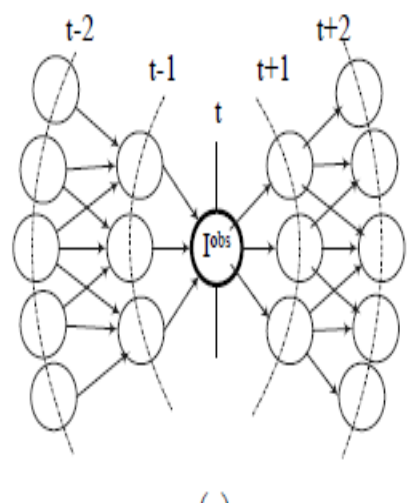

(a)

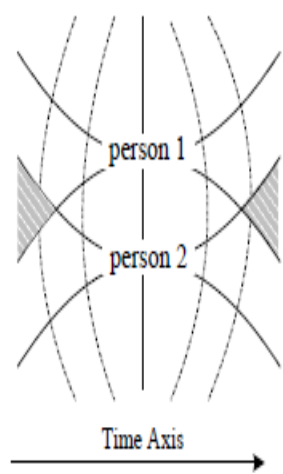

(b)
Fig 3: diagram of proposed approach

A dynamic model for face aging is implemented successfully.

\subsection{An Associate-Predict Model for Face Recognition [6]}

Now a day, in face recognition the most challenging task is how to handle intra-personal variation. Finding the similarity between human faces under various events like postures, lightning and utterance is very difficult. This paper proposed a contemporary system that tends to unravel this muddle, called "Associate-Predict" prediction model. Auxiliary generic identity data set acts as premise for the proposed model, having lots of person images with colossal intrapersonal variations. Separate facial parts like frontal and non-frontal are considered for the face recognition system. Firstly, one input face is "associated" with identical identities from the generic identities data set. On the basis of this face association generative "prediction" of the form for input face under the mount of another input faces or distinguish "prediction" of the prospect whether two faces that are input to the system belongs to the similar person or vice-versa. The prediction methods proposed are firstly "appearance-prediction" and secondly "likelihood-prediction "is used in this model. The intrapersonal variation is effectively controlled by another database called "memory" and "associate-predicate model". A switching mechanism is used to increase the generalized ability of this model. If two faces have adjacent intrapersonal mount then those faces can be directly compared else the proposed associate-predict model for the recognition is used. In this model, apart from the extra identities data set, restricted protocols are sternly followed, including Principal Component Analysis learning with Support Vector Machine based training of system. To show that this model can markedly ameliorate the performances of most subsisting face recognition methods experiments on two public face standards Multi-PIE and LFW are executed. 
Table 1: Comparison of various face prediction models

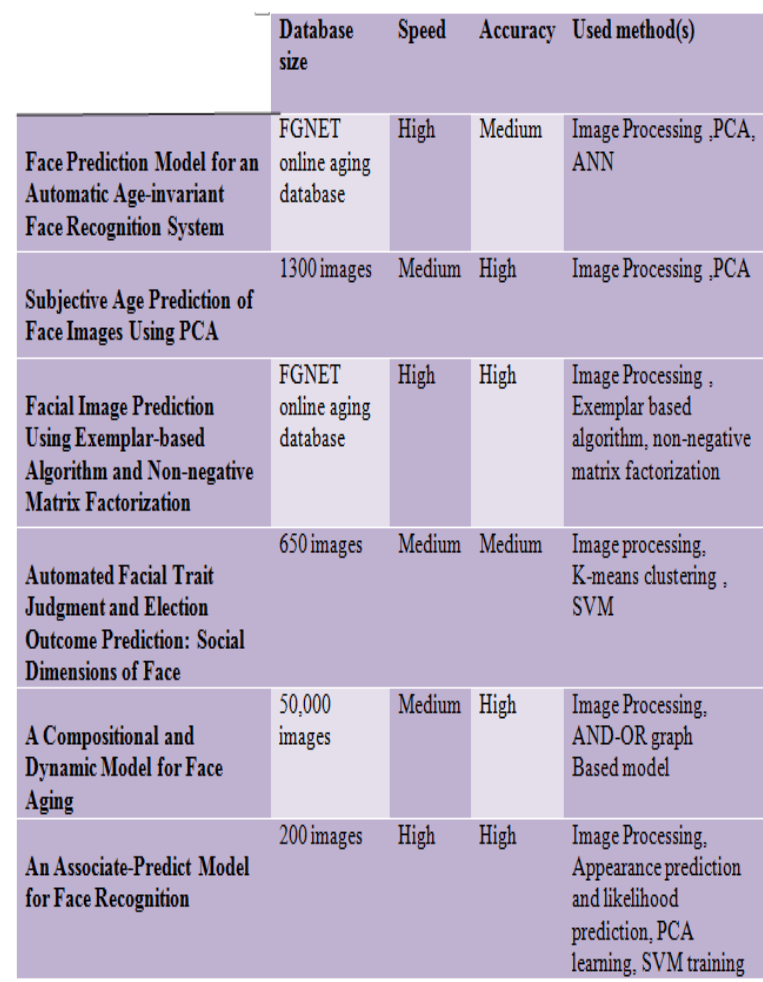

\section{CONCLUSION}

This paper gives a detailed description of various face prediction models by using image processing from which researcher can get an idea for an efficient face prediction techniques considering aging affects on human faces. This paper has comparison made between existing models on the basis of accuracy, speed, used methods, database size. There are future scopes of improvements in present methodologies as no model guarantee hundred percent accuracy and are restrained to specific feature. Present methodologies can be expanded by combining face prediction and recognition, so that a robust recognition based prediction model can be developed keeping in purview the effects of aging, facial traits, environment etc that is capable of recognizing current input image that is not present in the image database by predicting it on the basis of previously stored images that corresponding to current input image.

\section{REFERENCES}

[1] Poonam Yadav face prediction model for an automatic age invariant face recognition system arXiv 1506.0604v1[cs.CV] 16 April 2015

[2] Hlaing Htake Khaung Tin Subjective Age Prediction of Face Images Using PCA International Journal of Information and Electronics Engineering, Vol. 2, No. 3, May 2012.

[3] Hsuan T. Chang and Hsiao W. Peng Facial Image Prediction Using Exemplar-based Algorithm and Nonnegative Matrix Factorization.

[4] Jungseock Joo, Francis F. Steen, and Song-Chun Zhu Automated Facial Trait Judgment and Election Outcome Prediction: Social Dimensions of Face.

[5] Jinli Suo, Song-Chun Zhu, Shiguang Shan and Xilin Chen A Compositional and Dynamic Model for Face
AgingJOURNAL OF LATEX CLASS FILES, VOL. *, NO. *, JANUARY 2009.

[6] Qi Yin, Xiaoou Tang, Jian Sun An Associate- Predict Model for Face Recognition.

[7] Kathryn Bonnen and Brendan Klare and Anil K. Jain,"Component-Based Representation in Automated Face Recognition," IEEE Transactions on Information Forensics and Security-Volume 8, pp 239-253, 2013.

[8] J. 1. Suo, Song-Chun Zhu, S. Shan, and X. Chen, "A Compositional and Dynamic Model for Face Aging," IEEE Transactions on PatternAnalysis and Machine Intelligence, vol. 32, no. 3, 2010.

[9] L. L. Shen, "Gabor Wavelet Selection and SVM Classification for Object Recognition," found online at www.sciencedirect.com, 2010.

[10] J. Bekios-Calfa, J. M. Buenaposada, and L. Baumela, "Revisiting Linear Discriminant Techniques in Gender Recognition," IEEETransactions on Pattern Analysis and Machine Intelligence, vol. 33, no. 4, 2011.

[11] H. Ai and G. Wei, "Face Gender Classification on Consumer Images in a Multiethnic Environment," in Proc. Conf. Advances in Biometrics, 2009.

[12] G. Guo, C. R. Dyer, Y. Fu, and T. S. Huang, "Is Gender Recognition Affected by Age?" in Proc. IEEE Int'l Conf. Computer VisionWorkshop Human-Computer Interaction, pp. 2032-2039, 2009.

[13] J. Zheng, and B. L. Lu, "A support vector machine classifier with automatic confidence and its application to gender classification," International Journal of Neurocomputing, pp. 1926-1935, 2011.

[14] K. Luu, T. Dai Bui, Y. Ching, Suen and K. Ricanek Jr, "Spectral Regression based Age Determination," 2010.

[15] Ms. Sonali. B. Maind, Ms. Priyanka Wankar Research Paper on Basic of Artificial Neural Network International Journal on Recent and Innovation Trends in Computing and Communication ISSN: 2321-8169 Volume: 2 Issue: 196 - 100 IJRITCC | January 2014, Available@ http://www.ijritcc.org.

[16] Dileep M R, Ajit Danti Two Level Decision for Human age prediction using Neural Network International Journal of Electronics Communication and Computer Technology (IJECCT) Volume 5 Issue ICICC (May 2015).

[17] Dileep M R and Ajit Danti, Structured Connectivity-Face model for the recognition of Human Facial Expressions, International Journal of Science and Applied Information Technology (IJSAIT), Vol. 3 , No.3, Pages : $01-07$ (2014) Special Issue of ICCET 2014, ISSN 2278-3083.

[18] M. J. Er, W. Chen, S. Wu, "High Speed Face Recognition based on discrete cosine transform and RBF neural network," IEEE Trans on Neural Network, vol. 16, No .3, pp. 679,691.

[19] K. Luu, T. Dai Bui, Y. Ching, Suen and K. Ricanek Jr, "Spectral Regression based Age Determination," 2010.

[20] G. Mallikarjuna Rao, G. R. Babu, G. V. Kumari, and N. K. Chaitanya, "Methodological Approach for Machine based Expression and Gender Classification," IEEE 
International Advance Computing Conference, pp.13691374, 2009.

[21] J. Zheng, and B. L. Lu, "A support vector machine classifier with automatic confidence and its application to gender classification," International Journal of Neurocomputing, pp. 1926-1935, 2011.

[22] Sithu Ubaid, Dr. Shyama Das, Imthiyas M.P. Human Age Prediction and Classification Using Facial ImageSithu Ubaid et.al / International Journal on Computer Science and Engineering (IJCSE) ISSN : 0975-3397Vol. 5 No. 05 May 2013.

[23] Gudong Guo, Yun Fu,Charles R. Dyer, Thomas S. Huang, "Image-Based Human Age Estimation by Manifold Learning and Locally Adjusted Robust Regression", IEEE Transaction On Image Processing, vol. 17, (2008).

[24] Shima Izadpanahi, Onsen Toygar, "Geometric FeatureBased Age Classification Using Facial Images", IET Conference on Image Processing, (2012).
[25] Hlaing Htake Khaung Tin, "Subjective Age Prediction of Face Images Using PCA", International Journal of Information and Electronics Engineering, vol. 2, no. 3, (2012).

[26] K. Luu, T. D. Bui, C. Y. Suen, K. Ricanek, "Combined local and holistic facial features for age determination",ICARCV, pp. 900-904, (2010).

[27] J. 1. Suo, Song-Chun Zhu, S. Shan, and X. Chen, "A Compositional and Dynamic Model for Face Aging", IEEE Transactions on Pattern Analysis and Machine Intelligence, vol. 32, no. 3, 2010.

[28] J. 1. Suo, Song-Chun Zhu, S. Shan, and X. Chen, "A Compositional and Dynamic Model for Face Aging", IEEE Transactions on Pattern Analysis and Machine Intelligence, vol. 32, no. 3, 2010.

[29] Sergio Verduci and Hanqi Zhuang A preliminary study on human face prediction. 